\title{
Efficient Synthesis of Polysubstituted Acylguanidines and Guanylureas
}

Alan R. Katritzky, * Boris V. Rogovoy, Xiaohong Cai, Nataliya Kirichenko, and Katherine V. Kovalenko

Center for Heterocyclic Compounds, Department of Chemistry, University of Florida, Gainesville, FL 32611-7200, USA

\section{katritzky@chem.ufl.edu}

\section{Supporting Information}

General. Melting points were determined using a capillary melting point apparatus equipped with a digital thermometer and are uncorrected. ${ }^{1} \mathrm{H}$ NMR $(300 \mathrm{MHz})$ and ${ }^{13} \mathrm{C}$ NMR $(75 \mathrm{MHz})$ spectra were recorded on 300 NMR spectrometer in $\mathrm{CDCl}_{3}$ (with TMS for ${ }^{1} \mathrm{H}$ and chloroform- $d$ for ${ }^{13} \mathrm{C}$ as the internal reference). Optical rotation values were measured with the use of the sodium D line. Column chromatography was performed on silica gel (200-425 mesh), unless otherwise stated. All reactions were carried out under $\mathrm{N}_{2}$.

Characterization data for compounds $5 b-f$.

$N$-[1H-Benzotriazol-1-yl(diisopropylamino)methylidene]propanamide $\quad(5 b) . \quad$ White microcystals from methanol, mp 83-85 ${ }^{\circ} \mathrm{C} ;{ }^{1} \mathrm{H}$ NMR $\delta 0.93(\mathrm{t}, J=7.4 \mathrm{~Hz}, 3 \mathrm{H}), 1.43(\mathrm{~d}, J=6.7$ $\mathrm{Hz}, 12 \mathrm{H}), 2.26(\mathrm{q}, J=7.4 \mathrm{~Hz}, 2 \mathrm{H}), 3.54-3.63(\mathrm{~m}, 2 \mathrm{H}), 7.38-7.44(\mathrm{~m}, 1 \mathrm{H}), 7.55-7.57(\mathrm{~m}, 2 \mathrm{H})$, 
$8.08(\mathrm{~d}, J=8.4 \mathrm{~Hz}, 1 \mathrm{H}) ;{ }^{13} \mathrm{C}$ NMR $\delta 9.2,20.5,32.7,49.6,110.2,120.3,124.7,128.9,133.1$, 142.4, 145.0, 183.7. Anal. Calcd for $\mathrm{C}_{16} \mathrm{H}_{23} \mathrm{~N}_{5} \mathrm{O}: \mathrm{C}, 63.76 ; \mathrm{H}, 7.69 ; \mathrm{N}, 23.24$. Found: C, 64.08; H, $7.83 ; \mathrm{N}, 23.38$.

$N$-[1H-Benzotriazol-1-yl(diisopropylamino)methylidene]benzamide (5c). White flakes from methanol; mp $116-118{ }^{\circ} \mathrm{C}\left(\right.$ lit. $\left.^{24 a} 116-117{ }^{\circ} \mathrm{C}\right) ;{ }^{1} \mathrm{H}$ NMR $\delta 1.53(\mathrm{~d}, J=6.6 \mathrm{~Hz}, 12 \mathrm{H})$, 3.65-3.82 (m, 2H), 7.26-7.50 (m, 6H), 7.90-8.10 (m, 3H); ${ }^{13} \mathrm{C}$ NMR $\delta 20.7,50.2,110.2,120.4$, 124.6, 128.0, 128.8, 129.4, 131.9, 133.0, 136.0, 145.1, 145.8, 173.8.

$N$-[1H-Benzotriazol-1-yl(morpholino)methylidene]-4-methoxybenzamide $\quad(5 \mathrm{~d}) . \quad$ White microcrystals from chloroform/hexanes; mp $198-200{ }^{\circ} \mathrm{C} ;{ }^{1} \mathrm{H}$ NMR (DMSO- $d_{6}$ ) $\delta 3.61$ (br s, 4H), 3.80-3.82 (m, 7H), $6.98(\mathrm{~d}, J=8.5 \mathrm{~Hz}, 2 \mathrm{H}), 7.50(\mathrm{t}, J=7.1 \mathrm{~Hz}, 1 \mathrm{H}), 7.59-7.70(\mathrm{~m}, 2 \mathrm{H}), 7.91(\mathrm{~d}$, $J=8.4 \mathrm{~Hz}, 2 \mathrm{H}), 8.18(\mathrm{~d}, J=8.2 \mathrm{~Hz}, 1 \mathrm{H}) ;{ }^{13} \mathrm{C}$ NMR $\left(\right.$ DMSO- $\left.d_{6}\right) \delta 47.1,55.4,65.6,110.9,113.6$, 119.9, 125.2, 127.8, 129.6, 131.4, 132.3, 144.6, 145.8, 162.7, 173.6. Anal. Calcd for $\mathrm{C}_{19} \mathrm{H}_{19} \mathrm{~N}_{5} \mathrm{O}_{3}$ : C, 62.46; H, 5.24; N, 19.17. Found: C, 62.78; H, 5.22; N, 19.18.

$N$-(1H-Benzotriazol-1-yl(morpholino)methylidene)-2-furamide $\quad$ (5e). Colorless microcrystals from ethyl acetate, mp $146-147{ }^{\circ} \mathrm{C}$; ${ }^{1} \mathrm{H}$ NMR $\delta 3.67-3.70(\mathrm{~m}, 4 \mathrm{H}), 3.91-3.94(\mathrm{~m}$, 4H), 6.47-6.49 (m, 1H), $7.13(\mathrm{dd}, J=3.4 \mathrm{~Hz}, 0.7 \mathrm{~Hz}, 1 \mathrm{H}), 7.40-7.45(\mathrm{~m}, 1 \mathrm{H}), 7.49-7.59(\mathrm{~m}$, $3 \mathrm{H}), 8.09(\mathrm{~d}, J=8.4 \mathrm{~Hz}, 1 \mathrm{H}) ;{ }^{13} \mathrm{C}$ NMR $\delta 48.3,66.3,111.0,111.9,117.6,120.4,125.2,129.6$, 132.5, 145.5, 146.0, 147.7, 150.0, 165.4. Anal. Calcd for $\mathrm{C}_{16} \mathrm{H}_{15} \mathrm{~N}_{5} \mathrm{O}_{3}: \mathrm{C}, 59.07 ; \mathrm{H}, 4.65 ; \mathrm{N}, 21.53$. Found: C, 59.18; H, 4.53; N, 21.66.

$N$-[1H-Benzotriazol-1-yl(morpholino)methylidene]-4-chlorobenzamide $\quad(5 f) . \quad$ White microcrystals from methanol; mp 203-205 ${ }^{\circ} \mathrm{C} ;{ }^{1} \mathrm{H}$ NMR $\left(\right.$ DMSO- $\left.d_{6}\right) \delta 3.62-3.71(\mathrm{~m}, 4 \mathrm{H})$, 3.78-3.85 (m, 4H), 7.47-7.56 (m, 3H), 7.59-7.71 (m, 2H), $7.96(\mathrm{~d}, J=8.5 \mathrm{~Hz}, 2 \mathrm{H}), 8.19(\mathrm{~d}, J=$ 
$8.2 \mathrm{~Hz}, 1 \mathrm{H}) ;{ }^{13} \mathrm{C}$ NMR $\left(\right.$ DMSO- $\left.d_{6}\right) \delta 47.5,65.8,111.0,120.2,125.5,128.7,129.9,131.2,132.5$, 134.3, 137.6, 144.9, 147.0, 173.2. Anal. Calcd for $\mathrm{C}_{18} \mathrm{H}_{16} \mathrm{ClN}_{5} \mathrm{O}_{2}: \mathrm{C}, 58.46 ; \mathrm{H}, 4.36 ; \mathrm{N}, 18.94$. Found: C, 58.16; H, 4.32; N, 18.84 .

\section{Characterization data for compounds $7 \mathrm{~b}-0$.}

$N$-Morpholino(piperidino)methylidenebenzamide (7b). Microcrystals from ethyl acetate/hexanes, mp 96-97 ${ }^{\circ} \mathrm{C} ;{ }^{1} \mathrm{H}$ NMR $\delta$ Mixture of tautomers: $1.63(\mathrm{~s}, 6 \mathrm{H}), 3.29-3.40(\mathrm{~m}$, 8H), 3.72-3.75 $(\mathrm{m}, 4 \mathrm{H}), 7.39-7.47(\mathrm{~m}, 3 \mathrm{H}), 8.12-8.14(\mathrm{~m}, 2 \mathrm{H}) ;{ }^{13} \mathrm{C}$ NMR $\delta$ Mixture of tautomers described as appears on spectra. No distinction between the tautomeric forms is possible. $24.3,24.4,25.4,25.5,48.9,49.0,49.7,49.9,66.2,66.3,127.7,127.8,127.9,129.1$, 129.1, 129.2, 130.6, 130.9, 131.2, 137.0, 137.5, 137.9, 165.2, 166.4, 167.5, 173.5, 173.8, 174.1 . Anal. Calcd for $\mathrm{C}_{17} \mathrm{H}_{23} \mathrm{~N}_{3} \mathrm{O}_{2}: \mathrm{C}, 67.75 ; \mathrm{H}, 7.69 ; \mathrm{N}, 13.94$. Found: $\mathrm{C}, 67.50 ; \mathrm{H}, 7.71 ; \mathrm{N}, 13.80$.

$N$-Anilino(piperidino)methylidenebenzamide (7c). Microcrystals from AcOEt (76\%), mp 149-151 ${ }^{\circ} \mathrm{C} ;{ }^{1} \mathrm{H}$ NMR $\delta 1.61(\mathrm{~s}, 6 \mathrm{H}), 3.51-3.53(\mathrm{~m}, 4 \mathrm{H}), 7.05-7.10(\mathrm{~m}, 3 \mathrm{H}), 7.31$ (t, $J=7.9 \mathrm{~Hz}$, 2H), 7.38-7.47 (m, 3H), $8.24(\mathrm{~d}, J=6.9 \mathrm{~Hz}, 2 \mathrm{H}), 11.78(\mathrm{~s}, 1 \mathrm{H}) ;{ }^{13} \mathrm{C}$ NMR $\delta$ 24.3, 25.6, 48.0, 121.1, 124.1, 127.8, 129.1, 129.3, 131.2, 138.4, 140.0, 159.5, 176.7. Anal. Calcd for $\mathrm{C}_{19} \mathrm{H}_{21} \mathrm{~N}_{3} \mathrm{O}$ : C, 74.24; H, 6.89; N, 13.67. Found: C, 73.90; H, 7.03; N, 13.51.

$N$-(Butylamino)(piperidino)methylidenebenzamide (7d). White prisms from ethyl acetate, mp $162-163{ }^{\circ} \mathrm{C} ;{ }^{1} \mathrm{H}$ NMR $\delta 0.91(\mathrm{t}, J=7.3 \mathrm{~Hz}, 3 \mathrm{H}), 1.35-1.42(\mathrm{~m}, 2 \mathrm{H}), 1.55-1.66(\mathrm{~m}, 2 \mathrm{H}), 1.66$ (s, 6H), 3.20-3.26 (m, 2H), 3.50 (s, 4H), 7.35-7.43 (m, 3H), 8.18-8.21 (m, 2H), 9.07 (br s, 1H); ${ }^{13} \mathrm{C}$ NMR $\delta 13.7,20.0,24.4,25.8,32.4,45.2,48.1,127.7,128.9,130.6,138.8,164.5,174.9$. Anal. Calcd for $\mathrm{C}_{17} \mathrm{H}_{25} \mathrm{~N}_{3} \mathrm{O}: \mathrm{N}, 14.62$. Found: $\mathrm{N}, 14.63$.

$N, N$-Diisopropyl- $N$ '-phenethyl- $N^{\prime \prime}$-propionylguanidine (7e). Microcrystals from methanol, mp 96-98 ${ }^{\circ} \mathrm{C} ;{ }^{1} \mathrm{H}$ NMR $\delta 1.14-1.21(\mathrm{~m}, 15 \mathrm{H}), 2.32(\mathrm{q}, J=7.6 \mathrm{~Hz}, 2 \mathrm{H}), 2.90(\mathrm{t}, J=6.7 \mathrm{~Hz}, 2 \mathrm{H})$, 
$3.53(\mathrm{t}, J=6.7 \mathrm{~Hz}, 2 \mathrm{H}), 3.90-4.00(\mathrm{~m}, 2 \mathrm{H}), 5.20(\mathrm{br} \mathrm{s}, 1 \mathrm{H}), 7.22-7.34(\mathrm{~m}, 5 \mathrm{H}) ;{ }^{13} \mathrm{C}$ NMR $\delta 11.1$, 20.7, 32.6, 35.1, 43.9, 47.1, 126.8, 128.8, 138.2, 161.6, 178.4. Anal. Calcd for $\mathrm{C}_{18} \mathrm{H}_{29} \mathrm{~N}_{3} \mathrm{O}: \mathrm{C}$, 71.25; H, 9.63; N, 13.85. Found: C, 71.32; H, 9.95; N, 13.84.

$N^{\prime}$-Benzyl- $N, N$-diisopropyl- $N^{\prime \prime}$-propionylguanidine (7f). Microcrystals from methanol (50\%), mp 105-106 ${ }^{\circ} \mathrm{C} ;{ }^{1} \mathrm{H}$ NMR $\delta 1.19(\mathrm{t}, J=7.6 \mathrm{~Hz}, 3 \mathrm{H}), 3.29(\mathrm{~d}, J=6.9 \mathrm{~Hz}, 12 \mathrm{H}), 2.35(\mathrm{q}, J$ $=7.6 \mathrm{~Hz}, 2 \mathrm{H}), 3.98-4.07(\mathrm{~m}, 2 \mathrm{H}), 438(\mathrm{~s}, 2 \mathrm{H}), 2.64(\mathrm{br} \mathrm{s}, 1 \mathrm{H}), 7.30-7.36(\mathrm{~m}, 5 \mathrm{H}) ;{ }^{13} \mathrm{C} \mathrm{NMR} \delta$ 10.9, 21.0, 32.5, 47.4, 47.6, 127.7, 128.0, 128.7, 137.4, 161.2, 178.6. Anal. Calcd for $\mathrm{C}_{17} \mathrm{H}_{27} \mathrm{~N}_{3} \mathrm{O}$ : C, 70.55; H, 9.40; N, 14.52. Found: C, 70.39; H, 9.67; N, 14.52.

$N, N$-Diisopropyl- $N^{\prime}$-isobutyl- $N^{\prime \prime}$-propionylguanidine $\mathbf{( 7 g )}$. White microcrystals from diethyl ether/hexanes, mp 95- $97{ }^{\circ} \mathrm{C} ;{ }^{1} \mathrm{H}$ NMR $\delta 0.95(\mathrm{~d}, J=6.8 \mathrm{~Hz}, 6 \mathrm{H}), 1.17(\mathrm{t}, J=7.6 \mathrm{~Hz}, 3 \mathrm{H}), 1.32$ $(\mathrm{d}, J=6.8 \mathrm{~Hz}, 12 \mathrm{H}), 1.78-1.90(\mathrm{~m}, 1 \mathrm{H}), 2.31(\mathrm{q}, J=7.6 \mathrm{~Hz}, 2 \mathrm{H}), 3.02(\mathrm{~d}, J=6.8 \mathrm{~Hz}, 1 \mathrm{H})$, 3.90-4.04 (m, 2H); ${ }^{13} \mathrm{C}$ NMR $\delta 11.1,20.3,21.1,28.4,32.6,47.4,51.0,162.4$, 178.8. Anal. Calcd for $\mathrm{C}_{14} \mathrm{H}_{29} \mathrm{~N}_{3} \mathrm{O}$ : C, 65.84; H, 11.44; N, 16.45. Found: C, 66.18; H, 12.01; N, 16.51.

$N, N$-Diisopropyl- $N^{\prime}$-(4-methoxybenzyl)- $N^{\prime \prime}$-propionylguanidine (7h). White microcrystals from diethyl ether/hexanes; mp $124-125{ }^{\circ} \mathrm{C} ;{ }^{1} \mathrm{H}$ NMR $\delta 1.20(\mathrm{t}, J=7.6 \mathrm{~Hz}, 3 \mathrm{H}), 1.28(\mathrm{~d}, J=6.8$ $\mathrm{Hz}, 12 \mathrm{H}), 2.35(\mathrm{q}, J=7.6 \mathrm{~Hz}, 2 \mathrm{H}), 3.80(\mathrm{~s}, 3 \mathrm{H}), 3.96-4.07(\mathrm{~m}, 2 \mathrm{H}), 4.30(\mathrm{~d}, J=4.4 \mathrm{~Hz}, 2 \mathrm{H})$, $5.41($ br s, $1 \mathrm{H}), 6.88(\mathrm{~d}, J=8.5 \mathrm{~Hz}, 2 \mathrm{H}), 7.25(\mathrm{~d}, J=8.5 \mathrm{~Hz}, 2 \mathrm{H}) ;{ }^{13} \mathrm{C}$ NMR $\delta 11.1,21.1,32.8$, 47.2, 47.3, 55.2, 114.1, 129.5, 129.6, 159.2, 161.2, 178.9. Anal. Calcd for $\mathrm{C}_{18} \mathrm{H}_{29} \mathrm{~N}_{3} \mathrm{O}_{2}: \mathrm{C}, 67.68$; H, 9.15; N, 13.15. Found: C, 67.89; H, 9.55; N, 13.07.

$N^{\prime \prime}$-Benzoyl- $N^{\prime}$-phenyl- $N, N$-diisopropylguanidine (7i). White prisms from methanol; mp 141-143 ${ }^{\circ} \mathrm{C} ;{ }^{1} \mathrm{H}$ NMR $\delta 1.34(\mathrm{~d}, J=6.7 \mathrm{~Hz}, 12 \mathrm{H}), 3.80-3.90(\mathrm{~m}, 2 \mathrm{H}), 7.02-7.12(\mathrm{~m}, 3 \mathrm{H}), 7.28$ $(\mathrm{t}, J=7.5 \mathrm{~Hz}, 2 \mathrm{H}), 7.38-7.50(\mathrm{~m}, 3 \mathrm{H}), 8.22(\mathrm{~d}, J=7.5 \mathrm{~Hz}, 2 \mathrm{H}), 11.14(\mathrm{br}, 1 \mathrm{H}) ;{ }^{13} \mathrm{C}$ NMR $\delta$ 
21.0, 48.8, 122.8, 124.6, 127.8, 129.1, 129.3, 131.0, 138.6, 141.3, 160.5, 175.6. Anal. Calcd for $\mathrm{C}_{20} \mathrm{H}_{25} \mathrm{~N}_{3} \mathrm{O}: \mathrm{C}, 74.27 ; \mathrm{H}, 7.79 ; \mathrm{N}, 12.99$. Found: $\mathrm{C}, 74.35 ; \mathrm{H}, 8.04 ; \mathrm{N}, 13.13$.

$N^{\prime \prime}$-Benzoyl- $N^{\prime}$-benzyl- $N, N$-diisopropylguanidine $(\mathbf{7 j})$. White microcrystals from diethyl ether $/ \mathrm{CH}_{2} \mathrm{Cl}_{2} ; \operatorname{mp~} 163-165{ }^{\circ} \mathrm{C} ;{ }^{1} \mathrm{H}$ NMR $\delta 1.34(\mathrm{~d}, J=7.9 \mathrm{~Hz}, 12 \mathrm{H}), 4.01-4.10(\mathrm{~m}, 2 \mathrm{H}), 4.41(\mathrm{~d}$, $J=4.8 \mathrm{~Hz}, 2 \mathrm{H}), 5.92($ br s, $1 \mathrm{H}), 7.27-7.43(\mathrm{~m}, 8 \mathrm{H}), 8.14-8.20(\mathrm{~m}, 2 \mathrm{H}) ;{ }^{13} \mathrm{C}$ NMR $\delta 21.2,47.7$, 48.0, 127.6, 127.7, 128.1, 128.8, 128.9, 130.1, 137.6, 138.7, 162.4, 170.1. Anal. Calcd for $\mathrm{C}_{21} \mathrm{H}_{27} \mathrm{~N}_{3} \mathrm{O}: \mathrm{C}, 74.74 ; \mathrm{H}, 8.06 ; \mathrm{N}, 12.45$. Found: $\mathrm{C}, 74.95 ; \mathrm{H}, 8.18 ; \mathrm{N}, 12.61$.

$N$-(Dimorpholinomethylene)-4-methoxybenzamide (7k). White prisms from diethyl ether $/ \mathrm{CH}_{2} \mathrm{Cl}_{2}$; mp $159-161{ }^{\circ} \mathrm{C} ;{ }^{1} \mathrm{H}$ NMR $\delta 3.34(\mathrm{t}, J=4.5 \mathrm{~Hz}, 8 \mathrm{H}), 3.73(\mathrm{t}, J=4.4 \mathrm{~Hz}, 8 \mathrm{H}), 3.85$ $(\mathrm{s}, 3 \mathrm{H}), 6.90(\mathrm{~d}, J=8.8 \mathrm{~Hz}, 2 \mathrm{H}), 8.08(\mathrm{~d}, J=8.8 \mathrm{~Hz}, 2 \mathrm{H}) ;{ }^{13} \mathrm{C} \mathrm{NMR} \delta$ 49.0, 55.3, 66.2, 113.0, 129.7, 131.2, 162.2, 164.6, 174.0. Anal. Calcd for $\mathrm{C}_{17} \mathrm{H}_{23} \mathrm{~N}_{3} \mathrm{O}_{4}: \mathrm{C}, 61.25 ; \mathrm{H}, 6.95 ; \mathrm{N}, 12.60$. Found: C, 61.56; H, 7.14; N, 12.69 .

$N$-Morpholino(phenethylamino)methylidene-2-furamide (71). Colorless oil. ${ }^{1} \mathrm{H}$ NMR $\delta 2.88$ (t, $J=7.1 \mathrm{~Hz}, 2 \mathrm{H}), 3.45-3.49(\mathrm{~m}, 6 \mathrm{H}), 3.66-3.69(\mathrm{~m}, 4 \mathrm{H}), 6.45-6.46(\mathrm{~m}, 1 \mathrm{H}), 7.05(\mathrm{~d}, J=3.4 \mathrm{~Hz}$, 1H), 7.16-7.31 (m, 5H), $7.51(\mathrm{~s}, 1 \mathrm{H}), 8.33$ (br s, 1H). ${ }^{13} \mathrm{C}$ NMR $\delta 36.2,46.3,46.7,66.2,111.3$, 114.3, 126.7, 128.6, 128.7, 138.1, 144.4, 152.2, 163.3, 165.5. Anal. Calcd for $\mathrm{C}_{18} \mathrm{H}_{21} \mathrm{~N}_{3} \mathrm{O}_{3}: ; \mathrm{N}$, 12.83. Found: N, 12.60 .

$N$-Morpholino(4-toluidino)methylidene-2-furamide (7m). Microcrystals from methanol, mp 139-140 ${ }^{\circ} \mathrm{C} ;{ }^{1} \mathrm{H}$ NMR $\delta 2.32$ (s, 3H), 3.50-3.53 (m, 4H), 3.64-3.67 (m, 4H), 6.46-6.48 (m, 1H), $6.98(\mathrm{~d}, J=8.4 \mathrm{~Hz}, 2 \mathrm{H}), 7.12-7.14(\mathrm{~m}, 3 \mathrm{H}), 7.53-7.54(\mathrm{~m}, 1 \mathrm{H}), 11.47(\mathrm{br} \mathrm{s}, 1 \mathrm{H}) ;{ }^{13} \mathrm{C}$ NMR $\delta$ 20.8, 47.0, 66.3, 111.5, 115.3, 121.7, 130.1, 134.6, 136.6, 145.1, 152.2, 159.7, 168.5. Anal. Calcd for $\mathrm{C}_{17} \mathrm{H}_{19} \mathrm{~N}_{3} \mathrm{O}_{3}: \mathrm{C}, 65.16 ; \mathrm{H}, 6.11 ; \mathrm{N}, 13.41$. Found: C, 64.81; H, 6.18; N, 13.39. 
Methyl 2-\{[[(4-Chlorobenzoyl)imino](morpholino)methyl $]$ amino $\}-2-p h e n y l a c e t a t e$

Hydrochloride (7n). White prisms from methanol; mp $102-104{ }^{\circ} \mathrm{C} ;{ }^{1} \mathrm{H}$ NMR $\delta 3.44-3.50(\mathrm{~m}$, 4H), 3.69 (s, 3H), 3.74 (t, J = 4.8 Hz, 4H), 4.69 (br s, 1H), 5.37 (s, 1H), 7.28-7.33 (m, 7H), 7.96 $(\mathrm{d}, J=8.5 \mathrm{~Hz}, 2 \mathrm{H}) ;{ }^{13} \mathrm{C} \mathrm{NMR} \delta 46.8,53.0,60.4,66.1,127.1,127.9,128.8,129.0,130.6,135.6$, 136.2, 137.0, 160.9, 171.1, 171.7. Anal. Calcd for $\mathrm{C}_{21} \mathrm{H}_{23} \mathrm{Cl}_{2} \mathrm{~N}_{3} \mathrm{O}_{4}: \mathrm{C}, 55.76 ; \mathrm{H}, 5.13 ; \mathrm{N}, 9.29$. Found: C, 55.42; H, 4.62; N, 9.57.

4-Chloro- $N$-[(4-methoxyanilino)(morpholino)methylidene $]$ benzamide (7o). White prisms from methanol; mp $121-123{ }^{\circ} \mathrm{C} ;{ }^{1} \mathrm{H}$ NMR $\delta 3.53(\mathrm{t}, J=4.6 \mathrm{~Hz}, 4 \mathrm{H}), 3.67(\mathrm{t}, J=4.6 \mathrm{~Hz}, 4 \mathrm{H})$, $3.80(\mathrm{~s}, 3 \mathrm{H}), 6.87(\mathrm{~d}, J=6.9 \mathrm{~Hz}, 2 \mathrm{H}), 7.05(\mathrm{~d}, J=6.9 \mathrm{~Hz}, 2 \mathrm{H}), 7.37(\mathrm{~d}, J=8.5 \mathrm{~Hz}, 2 \mathrm{H}), 8.14(\mathrm{~d}$, $J=8.5 \mathrm{~Hz}, 2 \mathrm{H}), 11.8$ (br s, $1 \mathrm{H}) ;{ }^{13} \mathrm{C}$ NMR $\delta 47.1,55.4,66.3,114.7,123.4,128.1,130.5,132.0$, 136.7, 137.5, 157.0, 160.1, 175.7. Anal. Calcd for $\mathrm{C}_{19} \mathrm{H}_{20} \mathrm{ClN}_{3} \mathrm{O}_{3}: \mathrm{C}, 61.04 ; \mathrm{H}, 5.39 ; \mathrm{N}, 11.24$. Found: C, 61.05; H, 5.39; N, 11.19.

\section{Characterization data for compounds 11b-d.}

$N$-[1H-Benzotriazol-1-yl(benzylamino)methylidene]- $N^{\prime}$-(4-methoxyphenyl)urea

(11b).

White needles (76\%), mp 133-134 ${ }^{\circ} \mathrm{C} ;{ }^{1} \mathrm{H}$ NMR $\delta 3.78(\mathrm{~s}, 3 \mathrm{H}), 4.86(\mathrm{~d}, J=5.5 \mathrm{~Hz}, 2 \mathrm{H}), 6.86(\mathrm{~d}$, $J=8.9 \mathrm{~Hz}, 2 \mathrm{H}), 7.25-7.31(\mathrm{~m}, 5 \mathrm{H}), 7.39-7.46(\mathrm{~m}, 4 \mathrm{H}), 7.57(\mathrm{t}, J=7.6 \mathrm{~Hz}, 1 \mathrm{H}), 8.10(\mathrm{t}, J=8.2$ $\mathrm{Hz}, 2 \mathrm{H}), 10.40-10.90$ (m, 1H).; ${ }^{13} \mathrm{C}$ NMR $\delta 49.1,55.4,114.2,120.0,121.0,125.3,127.5,127.9$, 128.8, 129.3, 131.4, 132.0, 136.5, 145.7, 156.0, 163.8, 168.3. Anal. Calcd for $\mathrm{C}_{22} \mathrm{H}_{20} \mathrm{~N}_{6} \mathrm{O}_{2}: \mathrm{C}$, 65.99; H, 5.03; N, 20.99. Found: C, 66.15; H, 5.08; N, 21.03.

$N$-[1H-Benzotriazol-1-yl(4-toluidino)methylidene]- $N^{\prime}$-phenylurea (11c). Microcrystals (73\%), mp 126-127 ${ }^{\circ} \mathrm{C} ;{ }^{1} \mathrm{H}$ NMR $\delta 2.24(\mathrm{~s}, 3 \mathrm{H}), 7.04-7.10(\mathrm{~m}, 5 \mathrm{H}), 7.29(\mathrm{t}, J=7.4 \mathrm{~Hz}, 2 \mathrm{H})$, 7.45-7.53 (m, 1H), $7.65(\mathrm{~m}, 3 \mathrm{H}), 8.08(\mathrm{~d}, J=8.1 \mathrm{~Hz}, 1 \mathrm{H}), 8.20-8.28(\mathrm{~m}, 1 \mathrm{H}), 9.24$ (br s, $1 \mathrm{H})$, 
11.05 (br s, 1H); ${ }^{13} \mathrm{C}$ NMR $\delta 20.9,114.7,119.5,119.9,120.6,123.9,126.2,129.6,130.1,130.5$, 133.0, 136.2, 136.5, 140.5, 146.7, 148.9, 161.1. Anal. Calcd for $\mathrm{C}_{21} \mathrm{H}_{18} \mathrm{~N}_{6} \mathrm{O}: \mathrm{C}, 68.09 ; \mathrm{H}, 4.90 ; \mathrm{N}$, 22.69. Found: C, 67.99; H, 4.85; N, 22.47.

$N$-[1H-benzotriazol-1-yl(4-toluidino)methylidene]- $N^{\prime}$-(4-chlorophenyl)urea (11d). White microcrystals (89\%); ${ }^{1} \mathrm{H}$ NMR $\delta 2.29(\mathrm{~s}, 3 \mathrm{H}), 7.19(\mathrm{~d}, J=7.8 \mathrm{~Hz}, 2 \mathrm{H}), 7.26(\mathrm{~d}, J=8.6 \mathrm{~Hz}, 2 \mathrm{H})$, $7.33(\mathrm{~d}, J=8.6 \mathrm{~Hz}, 1 \mathrm{H}), 7.42(\mathrm{~d}, \mathrm{~J}=8.6 \mathrm{~Hz}, 2 \mathrm{H}), 7.48-7.57(\mathrm{~m}, 3 \mathrm{H}), 7.69(\mathrm{t}, J=7.8 \mathrm{~Hz}, 1 \mathrm{H})$ $8.21(\mathrm{~d}, J=8.2 \mathrm{~Hz}, 1 \mathrm{H}), 10.05(\mathrm{br} \mathrm{s}, 1 \mathrm{H}), 10.9(\mathrm{br} \mathrm{s}, 1 \mathrm{H}) ;{ }^{13} \mathrm{C}$ NMR $\delta 20.5,112.0,119.7,119.8$ 120.0, 121.4, 125.3, 125.5, 125.9, 128.3, 128.6, 129.3, 130.1, 131.8, 134.1, 138.5, 144.9, 152.3. This compound was used without purification for subsequent transformations.

\section{Characterization data for compounds 12b-h.}

$N-\left[\left(\right.\right.$ Hexylamino)(4-methoxyanilino)methylidene]- $N^{\prime}$-phenylurea hydrate (12b). Orange oil $(67 \%) ;{ }^{1} \mathrm{H}$ NMR $\delta 0.85-0.90(\mathrm{~m}, 3 \mathrm{H}), 1.25-1.35(\mathrm{~m}, 6 \mathrm{H}), 1.45-1.51(\mathrm{~m}, 2 \mathrm{H}), 3.30(\mathrm{dd}, J=$ 13.0, $6.8 \mathrm{~Hz}, 2 \mathrm{H}), 3.80(\mathrm{~s}, 3 \mathrm{H}), 4.50(\mathrm{br} \mathrm{s}, 1 \mathrm{H}), 6.91(\mathrm{~d}, J=8.8 \mathrm{~Hz}, 2 \mathrm{H}), 6.97(\mathrm{t}, J=7.4 \mathrm{~Hz}, 1 \mathrm{H})$, $7.14(\mathrm{~d}, J=8.8 \mathrm{~Hz}, 2 \mathrm{H}), 7.24-7.30(\mathrm{~m}, 3 \mathrm{H}), 7.48(\mathrm{~d}, J=7.8 \mathrm{~Hz}, 2 \mathrm{H}), 10.94(\mathrm{br} \mathrm{s}, 1 \mathrm{H}) . ;{ }^{13} \mathrm{C} \mathrm{NMR}$ $\delta 13.9,22.5,26.4,29.5,31.4,41.0,55.4,115.0,118.7,122.0,127.6,128.7,129.0,139.9,158.1$, 158.2, 163.6.. Anal. Calcd for $\mathrm{C}_{21} \mathrm{H}_{30} \mathrm{~N}_{4} \mathrm{O}_{3}: \mathrm{C}, 65.26 ; \mathrm{H}, 7.82 ; \mathrm{N}, 14.50$. Found: $\mathrm{C}, 65.17 ; \mathrm{H}, 7.59$; N, 14.23.

$N$-[(Benzylamino)(morpholino)methylidene]- $N^{\prime}$-(4-methoxyphenyl)urea hydrate (12c). Colorless oil (85\%); ${ }^{1} \mathrm{H}$ NMR $\delta$ 3.33-3.37 (m, 4H), 3.68-3.72 (4H), 3.77 (s, 3H), 4.40 (s, 2H), 6.81-6.85 (m, 2H), 7.26-7.38 (m, 8H), 9.40 (br s, 1H); ${ }^{13} \mathrm{C}$ NMR $\delta 47.9,48.9,55.4,66.4,114.0$, 120.7, 126.8, 127.5, 128.7, 130.8, 132.5, 137.7, 155.3, 163.9. Anal. Calcd for $\mathrm{C}_{20} \mathrm{H}_{26} \mathrm{~N}_{4} \mathrm{O}_{4}: \mathrm{C}$, 62.16; H, 6.78. Found: C, 62.30; H, 6.95. 
$N-\left[\left(\right.\right.$ Benzylamino)(butylamino)methylidene]- $N^{\prime}$-(4-methoxyphenyl)urea (12d). Colorless oil (87\%); ${ }^{1} \mathrm{H}$ NMR $\delta 0.89(\mathrm{t}, J=7.2 \mathrm{~Hz}, 3 \mathrm{H}), 1.24-1.34(\mathrm{~m}, 2 \mathrm{H}), 1.50$ (br s, 2H), 3.15 (br s, 2H), 3.77 (s, 3H), 4.48 (br s, 2H), $6.82(\mathrm{~d}, J=8.9 \mathrm{~Hz}, 2 \mathrm{H}), 6.84-7.00(\mathrm{~m}, 1 \mathrm{H}), 7.29-7.39(\mathrm{~m}$, 7H), 9.00-10.00 (m, 1H); ${ }^{13} \mathrm{C}$ NMR $\delta 13.6,19.9,31.2,40.7,45.0,55.4,113.9,114.3,120.8$, 127.2, 127.5, 128.8, 133.0, 155.0, 159.0, 163.7. HRMS Calcd for $\mathrm{C}_{20} \mathrm{H}_{26} \mathrm{~N}_{4} \mathrm{O}_{2}$ : 354.2056; Found: 354.2056.

$N$-(Benzylamino)(4-toluidino)methylidene- $N^{\prime}$-phenylurea hydrate (12e). Colorless oil $(60 \%) ;{ }^{1} \mathrm{H}$ NMR $\delta 1.62$ (br s, 1H), $2.33(\mathrm{~s}, 3 \mathrm{H}), 4.57$ (d, J= 5.2 Hz, 2H), 4.95 (br s, 1H), 6.99 (t, $J=7.4 \mathrm{~Hz}, 1 \mathrm{H}), 7.11(\mathrm{~d}, J=8.3 \mathrm{~Hz}, 2 \mathrm{H}), 7.17(\mathrm{~d}, J=8.3 \mathrm{~Hz}, 2 \mathrm{H}), 7.25-7.36(\mathrm{~m}, 7 \mathrm{H}), 7.48(\mathrm{~d}, J$ $=7.8 \mathrm{~Hz}, 2 \mathrm{H}), 11.11$ (br s, $1 \mathrm{H}) ;{ }^{13} \mathrm{C}$ NMR $\delta 20.9,44.8,118.9,122.3,125.7,127.3,127.4,128.7$, 128.8, 130.5, 133.6, 136.6, 138.6, 139.7, 157.7, 163.5. Anal. Calcd for $\mathrm{C}_{22} \mathrm{H}_{24} \mathrm{~N}_{4} \mathrm{O}_{2}: \mathrm{C}, 70.19 ; \mathrm{H}$, 6.43; Found: C, 69.89; H, 6.29.

$N$-[(Dibutylamino)(4-toluidino)methylidene]- $N^{\prime}$-phenylurea (12f). Colorless oil $(58 \%) ;{ }^{1} \mathrm{H}$ NMR $\delta 0.88(\mathrm{t}, J=7.3 \mathrm{~Hz}, 6 \mathrm{H}), 1.17-1.30(\mathrm{~m}, 4 \mathrm{H}), 1.45-1.55(\mathrm{~m}, 4 \mathrm{H}), 2.30(\mathrm{~s}, 3 \mathrm{H}), 3.22(\mathrm{t}, J=$ $7.3 \mathrm{~Hz}, 4 \mathrm{H}), 6.90-6.93(\mathrm{~m}, 2 \mathrm{H}), 6.94-7.00(\mathrm{~m}, 1 \mathrm{H}), 7.08(\mathrm{~d}, J=8.1 \mathrm{~Hz}, 2 \mathrm{H}), 7.22-7.38(\mathrm{~m}, 3 \mathrm{H})$, $7.47(\mathrm{~d}, J=8.1 \mathrm{~Hz}, 2 \mathrm{H}), 10.84($ br s, $1 \mathrm{H}) ;{ }^{13} \mathrm{C} \mathrm{NMR} \delta 13.8,20.1,20.7,29.7,48.0,118.8,121.7$, 122.2, 128.7, 129.8, 133.6, 138.1, 139.7, 160.1, 162.9. Anal. Calcd for $\mathrm{C}_{23} \mathrm{H}_{32} \mathrm{~N}_{4} \mathrm{O}: \mathrm{C}, 72.59 ; \mathrm{H}$, 8.48; N, 14.72. Found: C, 72.94; H, 8.79; N, 14.72 .

$N$-(4-Chlorophenyl)- $N^{\prime}$-[(dibutylamino)(4-toluidino)methylidene]urea (12g). Colorless oil (70\%); ${ }^{1} \mathrm{H}$ NMR $\delta$ mixture of tautomers 60:40 (according to ${ }^{1} \mathrm{H}$ NMR spectrum): $0.85-0.90$ $(0.93-0.98)(\mathrm{m}, 6 \mathrm{H}), 1.17-1.31(1.31-1.42)(\mathrm{m}, 4 \mathrm{H}), 1.45-1.55(1.57-1.65)(\mathrm{m}, 4 \mathrm{H}), 2.29(2.30)$ $(\mathrm{s}, 3 \mathrm{H}), 3.21(3.28)(\mathrm{t}, J=7.6 \mathrm{~Hz}, 4 \mathrm{H})$, signals of the two tautomers are indistinguishable and 
appear as described: 6.90-6.96 (m, 2H), 7.06-7.10 (m, 2H), 7.19-7.28 (m, 3H), 7.33-7.43 (m, 2H), 10.75 (10.82) (br s, 1H); ${ }^{13} \mathrm{C}$ NMR $\delta$ signals of the two tautomers are indistinguishable and appear as described: 13.7, 13.8, 20.0, 20.1, 20.7, 29.7, 30.7, 47.4, 48.0, 119.9, 120.9, 121.6, $121.7,126.9,127.5,128.5,128.6,129.1,129.2,129.7,129.8,133.7,137.9,138.0,138.4,154.6$, 160.1, 162.7. Anal. Calcd for $\mathrm{C}_{23} \mathrm{H}_{31} \mathrm{ClN}_{4} \mathrm{O}$ : C, 66.57; H, 7.53; N, 13.50. Found: C, 66.94; H, $7.90 ; \mathrm{N}, 13.74$.

$N$-[(4-Chloroanilino)(4-toluidino)methylene]- $N^{\prime}$-(4-chlorophenyl)urea $\quad(12 \mathrm{~h}) . \quad$ White microcrystals from ethyl acetate/hexanes (57\%), mp $174-175{ }^{\circ} \mathrm{C} ;{ }^{1} \mathrm{H}$ NMR (acetone- $d_{6}$ ) $\delta 2.33$ (s, 3H), 7.18-7.36 (m, 8H), 7.51-7.54 (m, 2H), 7.67-7.70 (m, 2H), 8.72 (br s, 1H), 8.82 (br s, $1 \mathrm{H}), 10.34$ (br s, $1 \mathrm{H}$ ); ${ }^{13} \mathrm{C}$ NMR (acetone- $\left.d_{6}\right) \delta 20.5,120.2,122.7,124.0,124.4,125.5,126.0$, 127.3, 128.4, 128.6, 129.5, 130.0, 133.3, 138.9, 174.5. Anal. Calcd for $\mathrm{C}_{21} \mathrm{H}_{18} \mathrm{Cl}_{2} \mathrm{~N}_{4} \mathrm{O}: \mathrm{C}, 61.03$; H, 4.39; N, 13.56. Found: C, 61.15; H, 4.45; N, 13.54.

\section{Characterization data for compounds 14e,f and 15b,d,e.}

$N$-[(4-Methoxybenzylamino)(dibenzylamino)methylidene]- $N$ '-phenylurea (14e). Colorless oil (35\%); ${ }^{1} \mathrm{H}$ NMR $\delta 3.76(\mathrm{~s}, 3 \mathrm{H}), 4.36(\mathrm{~d}, J=4.7 \mathrm{~Hz}, 2 \mathrm{H}), 4.46(\mathrm{~s}, 4 \mathrm{H}), 6.80(\mathrm{~d}, J=8.7 \mathrm{~Hz}$, 2H), $6.97(\mathrm{t}, J=7.3 \mathrm{~Hz}, 1 \mathrm{H}), 7.02(\mathrm{br} \mathrm{s}, 1 \mathrm{H}), 7.07-7.17(\mathrm{~m}, 6 \mathrm{H}), 7.21-7.34(\mathrm{~m}, 8 \mathrm{H}), 7.45(\mathrm{~d}, J=$ $7.9 \mathrm{~Hz}, 2 \mathrm{H}$ ), 8.68 (br s, $1 \mathrm{H}$ ); ${ }^{13} \mathrm{C}$ NMR $\delta 48.4,51.4,55.2,114.1,118.7,122.1,127.4,127.6$ 128.3, 128.6, 128.7, 130.0, 136.9, 139.8, 159.0, 162.0, 163.7. Anal. Calcd for $\mathrm{C}_{30} \mathrm{H}_{30} \mathrm{~N}_{4} \mathrm{O}_{2}: \mathrm{C}$, 75.29; H, 6.32; N, 11.71. Found: C, 74.87; H, 6.52; N, 11.84.

$N$-[(Butylamino)(dibenzylamino)methylidene]- $N^{\prime}$-phenylurea (14f). Colorless oil (31\%); ${ }^{1} \mathrm{H}$ NMR $\delta 0.88(\mathrm{t}, J=7.2 \mathrm{~Hz}, 3 \mathrm{H}), 1.30-1.40(\mathrm{~m}, 2 \mathrm{H}), 1.51-1.63(\mathrm{~m}, 2 \mathrm{H}), 3.20-3.28(\mathrm{~m}, 2 \mathrm{H}), 4.46$ (s, 4H), 6.88-7.02 (m, 2H), 7.17-7.38 (m, 12H), $7.45(\mathrm{~d}, J=7.9 \mathrm{~Hz}, 2 \mathrm{H}), 8.70($ br s, $1 \mathrm{H}) ;{ }^{13} \mathrm{C}$ 
NMR $\delta 13.5,19.8,32.4,45.3,51.1,118.6,121.9,127.2,127.6,128.5,128.6,137.1,139.8$ 162.3, 164.5. Anal. Calcd for $\mathrm{C}_{52} \mathrm{H}_{66} \mathrm{~N}_{8} \mathrm{O}_{5}: \mathrm{C}, 70.72 ; \mathrm{H}, 7.53 ; \mathrm{N}, 12.69$. Found: $\mathrm{C}, 70.97 ; \mathrm{H}, 7.14$; $\mathrm{N}, 12.41$.

$N$-Butyl- $N$ '-phenylurea (15b). White prisms from methanol, mp $130-132{ }^{\circ} \mathrm{C}$ (lit. ${ }^{30} 126-127$ $\left.{ }^{\circ} \mathrm{C}\right) ;{ }^{1} \mathrm{H}$ NMR $\delta 0.85(\mathrm{t}, \mathrm{J}=7.2 \mathrm{~Hz}, 3 \mathrm{H}), 1.18-1.44(\mathrm{~m}, 4 \mathrm{H}), 3.08-3.18(\mathrm{~m}, 2 \mathrm{H}), 5.84(\mathrm{br} \mathrm{s}, 1 \mathrm{H})$, $6.97(\mathrm{t}, \mathrm{J}=7.1 \mathrm{~Hz}, 1 \mathrm{H}), 7.16-7.29(\mathrm{~m}, 4 \mathrm{H}), 7.74(\mathrm{br} \mathrm{s}, 1 \mathrm{H}) ;{ }^{13} \mathrm{C}$ NMR $\delta 13.7,20.0,32.2,39.9$, $120.3,122.9,129.0,139.1,156.7$

$N$-Phenyl-4-morpholinecarboxamide (15d). White prisms from methanol (72\%), mp 156-159 ${ }^{\circ} \mathrm{C}\left(\right.$ lit. $\left.{ }^{31} 157-159{ }^{\circ} \mathrm{C}\right) ;{ }^{1} \mathrm{H}$ NMR $\left(\right.$ Acetone- $\left.d_{6}\right) \delta 3.47(\mathrm{t}, J=4.8 \mathrm{~Hz}, 4 \mathrm{H}), 3.62(\mathrm{t}, J=4.8$ $\mathrm{Hz}, 4 \mathrm{H}), 6.94(\mathrm{t}, J=7.3 \mathrm{~Hz}, 1 \mathrm{H}), 7.17-7.25(\mathrm{~m}, 2 \mathrm{H}), 7.49-7.55(\mathrm{~m}, 2 \mathrm{H}), 7.94(\mathrm{br} \mathrm{s}, 1 \mathrm{H}) ;{ }^{13} \mathrm{C}$ NMR (Acetone- $\left.d_{6}\right) \delta 45.3,67.2,120.4,122.9,129.3,141.5,156.2$. Anal. Calcd for $\mathrm{C}_{11} \mathrm{H}_{14} \mathrm{~N}_{2} \mathrm{O}_{2}$ : C, 64.06; H, 6.84; N, 13.58. Found: C, 64.48; H, 6.94; N, 13.62.

$N$-(4-Methoxybenzyl)- $N$ '-phenylurea (15e). White prisms from methanol (40\%), mp 161-163 ${ }^{\circ} \mathrm{C}$ (lit. $\left.{ }^{32} 160-162{ }^{\circ} \mathrm{C}\right) ;{ }^{1} \mathrm{H}$ NMR $\delta 3.72$ (s, 3H), 4.19 (d, J=5.6Hz, 2H), 5.77 (br s, $\left.1 \mathrm{H}\right)$, $6.75(\mathrm{~d}, J=8.5 \mathrm{~Hz}, 2 \mathrm{H}), 6.94-7.01(\mathrm{~m}, 1 \mathrm{H}), 7.10(\mathrm{~d}, J=8.5 \mathrm{~Hz}, 2 \mathrm{H}), 7.14-7.21(\mathrm{~m}, 4 \mathrm{H}) ;{ }^{13} \mathrm{C}$ NMR $\delta$ $43.5,55.2,113.9,120.6,123.3,128.6,129.1,131.0,138.7,156.2,158.8$. 